\title{
DIRECT DARK MATTER EVENT RATES WITH A VELOCITY DISTRIBUTION IN THE EDDINGTON APPROACH
}

\author{
J.D. Vergados ${ }^{1 *}$ and D. Owen ${ }^{2}$ \\ ${ }^{1}$ University of Ioannina, Ioannina, GR 45110, Greece ${ }^{\dagger}$. \\ 2 Department of Physics, Ben Gurion University, Israel ${ }^{\ddagger}$.
}

\begin{abstract}
Exotic dark matter together with the vacuum energy (associated with the cosmological constant) seem to dominate the Universe. Thus its direct detection is central to particle physics and cosmology. Supersymmetry provides a natural dark matter candidate, the lightest supersymmetric particle (LSP). One essential ingredient in obtaining the direct detection rates is the density and the velocity distribution of the LSP in our vicinity. In the present paper we study simultaneously density profiles and velocity distributions in the context of the Eddington approach. In such an approach, unlike the commonly assumed Maxwell-Boltzmann (M-B) distribution, the upper bound of the velocity arises naturally from the potential.
\end{abstract}

PACS numbers: $95.35 .+\mathrm{d}, 12.60 . \mathrm{Jv}$

\section{INTRODUCTION}

The combined MAXIMA-1 [1], BOOMERANG 2], DASI [3] and COBE/DMR Cosmic Microwave Background (CMB) observations [4] imply that the Universe is flat [5], $\Omega=1.11 \pm 0.07$ and that most of the matter in the Universe is Dark [6]. i.e. exotic. Combining the WMAP data with other experiments, crudely speaking one finds:

$$
\Omega_{b}=0.05, \Omega_{C D M}=0.30, \Omega_{\Lambda}=0.65
$$

Since the non exotic component cannot exceed $40 \%$ of the CDM [7], there is room for the exotic WIMP's (Weakly Interacting Massive Particles). Supersymmetry naturally provides candidates for the dark matter constituents [8]-[9]. In the most favored scenario of supersymmetry the LSP (Lightest Supersymmetric Particle) can be simply described as a Majorana fermion, a linear combination of the neutral components of the gauginos and higgsinos [8- [10]. In most calculations the neutralino is assumed to be primarily a gaugino, usually a bino. Even though there exists firm indirect evidence for a halo of dark matter in galaxies from the observed rotational curves, it is essential to directly detect [8]-[1] such matter. Until dark matter is actually detected, we shall not be able to exclude the possibility that the rotation curves result from a modification of the laws of nature as we currently view them. This makes it imperative that we invest a maximum effort in attempting to detect dark matter whenever it is possible. Furthermore such a direct detection will also unravel the nature of the constituents of dark matter.

The possibility of such detection, however, depends on the nature of the dark matter constituents (WIMPs). Since the WIMP is expected to be very massive, $m_{\chi} \geq 30 \mathrm{GeV}$, and extremely non relativistic with average kinetic energy $T \approx 50 \mathrm{KeV}\left(m_{\chi} / 100 \mathrm{GeV}\right)$, it can be directly detected [8]-[1] mainly via the recoiling of a nucleus $(\mathrm{A}, \mathrm{Z})$ in elastic scattering. The event rate for such a process can be computed from the following ingredients:

1. An effective Lagrangian at the elementary particle (quark) level obtained in the framework of supersymmetry as described, e.g., in Refs 10, 12.

2. A well defined procedure for transforming the amplitude obtained using the previous effective Lagrangian from the quark to the nucleon level, i.e. a quark model for the nucleon. This step is not trivial, since the obtained results depend crucially on the content of the nucleon in quarks

\footnotetext{
* Corresponding author

† e-mail: vergados@cc.uoi.gr

‡ e-mail: daowen137@gmail.com
} 
other than $\mathrm{u}$ and $\mathrm{d}$. This is particularly true for the scalar couplings, which are proportional to the quark masses [13]- 14], [15] as well as the isoscalar axial coupling [15, 16].

3. Knowledge of the relevant nuclear matrix elements [17]- 18], obtained with as reliable as possible many body nuclear wave functions. Fortunately in the case of the scalar coupling, which is viewed as the most important, the situation is a bit simpler, since then one needs only the nuclear form factor.

4. Knowledge of the WIMP density in our vicinity and its velocity distribution. Since the essential input here comes from the rotational curves, dark matter candidates other than the LSP (neutralino) are also characterized by similar parameters.

In the past various velocity distributions have been considered. The one most used is the isothermal Maxwell-Boltzmann velocity distribution with $\left\langle v^{2}\right\rangle=(3 / 2) v_{0}^{2}$ where $v_{0}$ is the velocity of the sun around the galaxy, i.e. $220 \mathrm{~km} / \mathrm{s}$. Extensions of this M-B distribution were also considered, in particular those that were axially symmetric with enhanced dispersion in the galactocentric direction 19, 20]. In such distributions an upper cutoff $v_{e s c}=2.84 v_{0}$ was introduced by hand.

Non isothermal models have also been considered. Among those one should mention the late infall of dark matter into the galaxy, i.e caustic rings [21, 22, 23, 24, 25], dark matter orbiting the Sun [26], Sagittarius dark matter [27].

The correct approach in our view is to consider the Eddington proposal 28], i.e. to obtain both the density and the velocity distribution from a mass distribution, which depends both on the velocity and the gravitational potential. Our motivation in using Eddington 28] approach to describing the density of dark matter is found, of course, in his success in describing the density of stars in globular clusters. Since this approach adequately describes the distribution of stars in a globular cluster in which the main interaction is gravitational and because of its generality, we see no reason why such an approach should not be applicable to dark matter that also interact gravitationally. It should be noted that the attempt to use Maxwellian (M-B) distribution to describe the star distribution in globular clusters led to results that did not correspond to observations [28]. So it seems that the use of M-B distribution to describe dark matter is not very well motivated and a different approach is required.

It seems, therefore, not surprising that this approach has been used by Merritt [29] and applied to dark matter by Ullio and Kamionkowski [30] and more recently by us [31]. It is the purpose of the present paper to obtain a dark matter velocity distribution, which is consistent with assumed halo matter distributions and has a natural upper velocity cut off. The results presented here are motivated by the dark matter candidate provided by supersymmetry, namely the LSP (neutralino). They can easily be extended, however, to be applied to other heavy WIMP candidates.

\section{THE DARK MATTER DISTRIBUTION IN THE CONTEXT OF THE EDDINGTON APPROACH}

As we have seen in the introduction the matter distribution can be given as follows

$$
d M=2 \pi f\left(\Phi(\mathbf{r}), v_{r}, v_{t}\right) d x d y d z v_{t} d v_{t} d v_{r}
$$

where the function $f$ the distribution function, which depends on $\mathbf{r}$ through the potential $\Phi(\mathbf{r})$ and the tangential and radial velocities $v_{t}$ and $v_{r}$. In general the distribution function is not symmetric. In the above expression we assumed that it is only axially symmetric, with the two tangential components being equal. Thus the density of matter $\rho$ satisfies the equation:

$$
d \rho=2 \pi f\left(\Phi(\mathbf{r}), v_{r}, v_{t}\right) \quad v_{t} d v_{t} d v_{r}
$$

It is more convenient instead of the velocities to use the total energy $E$ and the angular momentum $J$ via the equations

$$
J=v_{t} r \quad, \quad 2 E=v_{r}^{2}+\frac{J^{2}}{r^{2}}+2 \Phi(r)
$$


The use of these variables, which are constants of motion, is very useful, when one wants to study steady states. In doing this Eddington used the result of Jeans [32] that the density must be a function of first integrals of the equations of motion which follows from Liouville's theorem. The advantage of this approach is that the density can be 'inverted' and the velocity distribution can be found. Following this approach we find

$$
\rho=\frac{2 \pi}{r^{2}} \iint \frac{f(E, J) J}{\sqrt{2(E-\Phi(r))-J^{2} / r^{2}}} d J d E
$$

The limits of integration for $E$ are from $\Phi$ to 0 and for $J$ from 0 to $\left[2 r^{2}(E-\Phi(r))\right]^{1 / 2}$.

Furthermore if the distribution function is known one can obtain the velocity distribution at some point, e.g. in our vicinity, by

$$
\left.f\left(\Phi(\mathbf{r}), v_{r}, v_{t}\right)\right|_{\mathbf{r}=\mathbf{r}_{\mathbf{s}}}
$$

The problem which is more interesting is: Can one obtain the distribution function given the density (and hence the potential via Poisson's equation)? The answer is affirmative via Eddington's treatment of the distribution function and quite easy, if the distribution does not explicitly depend on $\mathrm{J}$, but is only a function of E. In the present work we will be concerned with spherically symmetric velocity distributions and we will leave out the more realistic axially symmetric case [33], 34]. Such axially symmetric velocity distributions have, however, been found to have interesting consequences on the direct dark matter detection rates, especially in directional experiments [35], [36].

If the angular momentum dependence is ignored, by integrating Eq. (4) one finds

$$
\rho=4 \pi \int f(E) \sqrt{2(E-\Phi(r))} d E
$$

with the range of $\mathrm{E}$ as above. In this case one can obtain the density as a function of the potential. Conversely if the density is given as a function of the potential one can proceed to find the distribution function according to the Eddington approach. The distribution then is a function of the total energy $E=v^{2} / 2+\Phi(r)$ and satisfies the Boltzmann Equation with the collision term zero, i.e.

$$
(\mathbf{v} \cdot \nabla \mathbf{r}-\nabla \Phi \cdot \nabla \mathbf{v}) f=0
$$

In this case the distribution can be expressed as follows:

$$
f(E)=\frac{\sqrt{2}}{4 \pi^{2}} \frac{d}{d E} \int_{E}^{0} \frac{d \Phi}{\sqrt{\Phi-E}} \frac{d \rho}{d \Phi}
$$

The above equation can be rewritten as:

$$
f(E)=\frac{1}{2 \sqrt{2} \pi^{2}}\left[\int_{E}^{0} \frac{d \Phi}{\sqrt{\Phi-E}} \frac{d^{2} \rho}{d \Phi^{2}}-\left.\frac{1}{\sqrt{-E}} \frac{d \rho}{d \Phi}\right|_{\Phi=0}\right]
$$

Thus we can obtain the distribution, if the density $\rho$ is given as a function of the potential $\Phi$. We find it convenient to rewrite the last equation in terms of dimensionless variables by introducing:

$$
\eta=\eta(\xi), \quad \eta=\frac{\rho}{\rho_{0}}, \quad \xi=\frac{\Phi}{\Phi_{0}}
$$

The constants $\rho_{0}$ and $\Phi_{0}$, which set the scale of these two quantities, are related through Poisson's equation. Then the Eddington distribution function takes the form:

$$
f(e)=\frac{\rho_{0}}{\pi^{2}\left|2 \Phi_{0}\right|^{3 / 2}}\left[\frac{\eta^{\prime}(0)}{\sqrt{e}}+\int_{0}^{e} \frac{\eta^{\prime \prime}(\xi)}{\sqrt{e-\xi}} d \xi\right]
$$

where $e$ is given by

$$
e=-\frac{E}{\left|\Phi_{0}\right|}=\xi-\frac{v^{2}}{2\left|\Phi_{0}\right|}
$$


i.e. $e$ the negative of the total energy $\epsilon(e>0$. In the presence of asymmetry one finds

$$
e=-\epsilon=\xi-\frac{1}{2\left|\Phi_{0}\right|}\left(v^{2}+\alpha_{s} v_{t}^{2}\right)
$$

where $\alpha_{s}$ is the asymmetry parameter, $v_{t}$ is the tangential velocity.

For numerical integrations it is more convenient to rewrite the last integral as follows:

$$
f(e)=\frac{\rho_{0}}{\pi^{2}\left|2 \Phi_{0}\right|^{3 / 2}}\left[\frac{\eta^{\prime}(0)}{\sqrt{e}}+2 \eta^{\prime \prime}(0) \sqrt{e}+2 \int_{0}^{e} \eta^{\prime \prime \prime}(\xi) \sqrt{e-\xi} d \xi\right]
$$

The first term is singular as $e \rightarrow 0$. This, however, causes no problem, since, as we have seen in our earlier work, the integrals over the velocity distribution are relevant in dark matter calculations and these remain finite as the velocity approaches the maximum velocity. Anyway in the example considered in the present work $\eta^{\prime}(0)=\eta^{\prime \prime}(0)=0$.

Once this function is known one obtains the velocity distribution of matter in our neighborhood $\left(r=r_{s}=0.8 a, a=\right.$ the galactic radius)with respect to the center of the galaxy via the relation

$$
f_{v}(v)=\left.f(e)\right|_{r=r_{s}=0.8 a},
$$

which must be normalized. The characteristic feature of this approach is that the velocity distribution vanishes outside a given region specified by a cut off velocity $v_{m}$, by setting $\left.e\right|_{r=r_{s}=0.8 a}=0$

\section{A SIMPLE REALISTIC DENSITY PROFILE}

We will consider three types of matter density:

- A spherical ordinary matter density (bulge density)

- Ordinary matter density in the form of a disc

- Dark matter density.

\section{A. Spherical ordinary matter density}

To obtain analytical expressions we will simulate this density as follows:

$$
\rho_{b}(x)=\rho_{0 b} \frac{\sqrt{2}}{\left(1+x^{2}\right)^{5 / 2}} \quad, \quad x=\frac{r}{a}
$$

with $\rho_{0 b}$ a constant and $a$ the galactic radius. So if this were the whole story, $\rho_{0 b}=2 \rho_{s}$ with $\rho_{s}$ the mass density in our vicinity. A distribution found by Plummer [37] and Von Zeipel 38], $\rho(x) \propto\left(1+x^{2}\right)^{-5 / 2}$ was obtained by considering a gas in spherical container with a specific heat ratio of $\gamma=1.2$. We note that a value of $\gamma=1$, which leads to isothermal distribution, was excluded by observation. This normal matter density profile is shown in Fig. 1] With this density one finds the potential:

$$
\Phi_{b}(x)=-4 \pi G_{N} a^{2} \rho_{0 b} \frac{\sqrt{2}}{6 \sqrt{x^{2}+1}}
$$

and the rotational velocity:

$$
v_{b}^{2}(x)=x \frac{d \Phi(x)}{d x}=4 \pi G_{N} a^{2} \rho_{0 b} \frac{\sqrt{2} x^{2}}{3\left(x^{2}+1\right)^{3 / 2}}
$$

The above potential and rotational velocity are shown in Fig. 2 


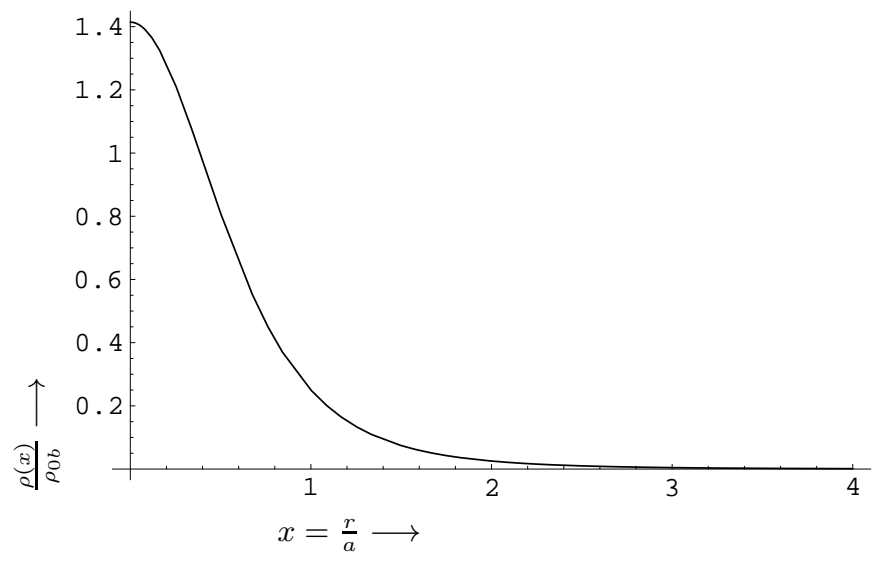

FIG. 1: The ordinary matter density distribution in dimensionless units.

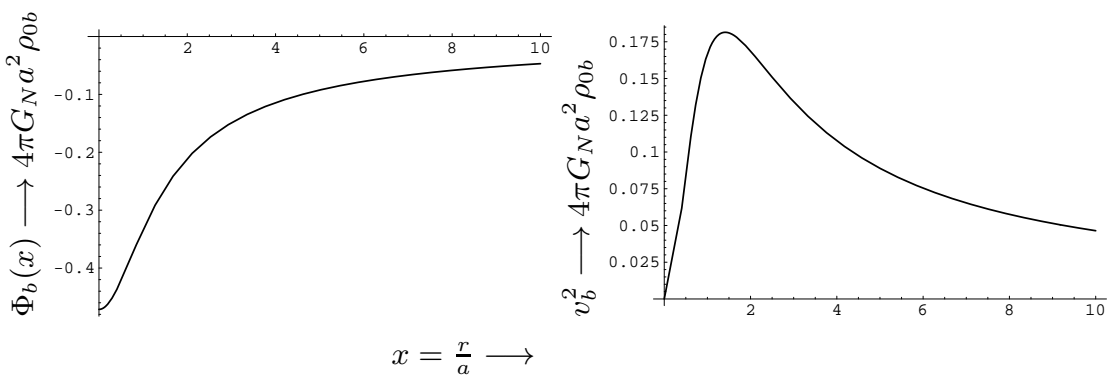

FIG. 2: The Potential and the rotational velocity in units of $4 \pi G_{N} a^{2} \rho_{0 b}$ due to the spherical ordinary matter distribution discussed in the text.

\section{B. Ordinary matter density distributed on a disc}

This is a more complicated problem. We will adopt the simplification that the matter distribution is a $\delta$ function along the axis of the disk. The radial density has been successfully modelled in the form of exponential profiles [39]. Nevertheless, to minimize the number of parameters employed, we will assume further that it has the same radial dependence as discussed above. In other words

$$
\rho_{d}(x, z)=\rho_{0 d} \delta(z) \frac{\sqrt{2}}{\left(1+x^{2}\right)^{5 / 2}} \quad, \quad x=\frac{r}{a}
$$

where $r$ is now the radial distance from the axis of symmetry (both $x$ and $z$ are measured in units of $a$ ). The potential now takes the form:

$$
\Phi_{d}(x, z)=4 \pi G_{N} a^{2} \rho_{0 d} \frac{1}{\pi} \int_{0}^{\infty} d k \cos (k z) G(x, k)
$$

where the Green's function in momentum space is given in terms of the modified Bessel functions:

$$
G(x, k)=G_{<}(x, k)+G_{>}(x, k)
$$

with

$$
\begin{aligned}
& G_{>}=-I_{0}(k x) \int_{x}^{\infty} K_{0}(k y) \rho_{b}(y) d y \\
& G_{<}=-K_{0}(k x) \int_{0}^{x} I_{0}(k y) \rho_{b}(y) d y
\end{aligned}
$$


The radial rotational velocity is given by

$$
v_{d}^{2}(x, z)=4 \pi G_{N} a^{2} \rho_{0 d} \frac{1}{\pi} x \int_{0}^{\infty} \cos (k z) g(x, k) d k
$$

with

$$
\begin{gathered}
g(x, k)=g_{<}(x, k)+g_{>}(x, k) \\
g_{<}=I_{0}(k x) K_{0}(k x) \rho_{d}(x)+k K_{1}(k x) \int_{0}^{x} I_{0}(k y) \rho_{d}(y) d y \\
g_{<}=I_{0}(k x) K_{0}(k x) \rho_{d}-k I_{1}(k x) \int_{x}^{\infty} K_{0}(k y) \rho_{d}(y) d y
\end{gathered}
$$

The obtained potential and the square of the rotational velocity on the plane of the galactic plane are shown in Fig. 3 In the same Figure we also plot the same quantity obtained with the exponential profile:

$$
\rho_{e d}(x, z)=\rho_{0 e d} \delta(z) e^{-3.5 x} \quad, \quad x=\frac{r}{a}
$$

We see that the two densities give essentially the same rotational velocities with the possible exception at very small distances.
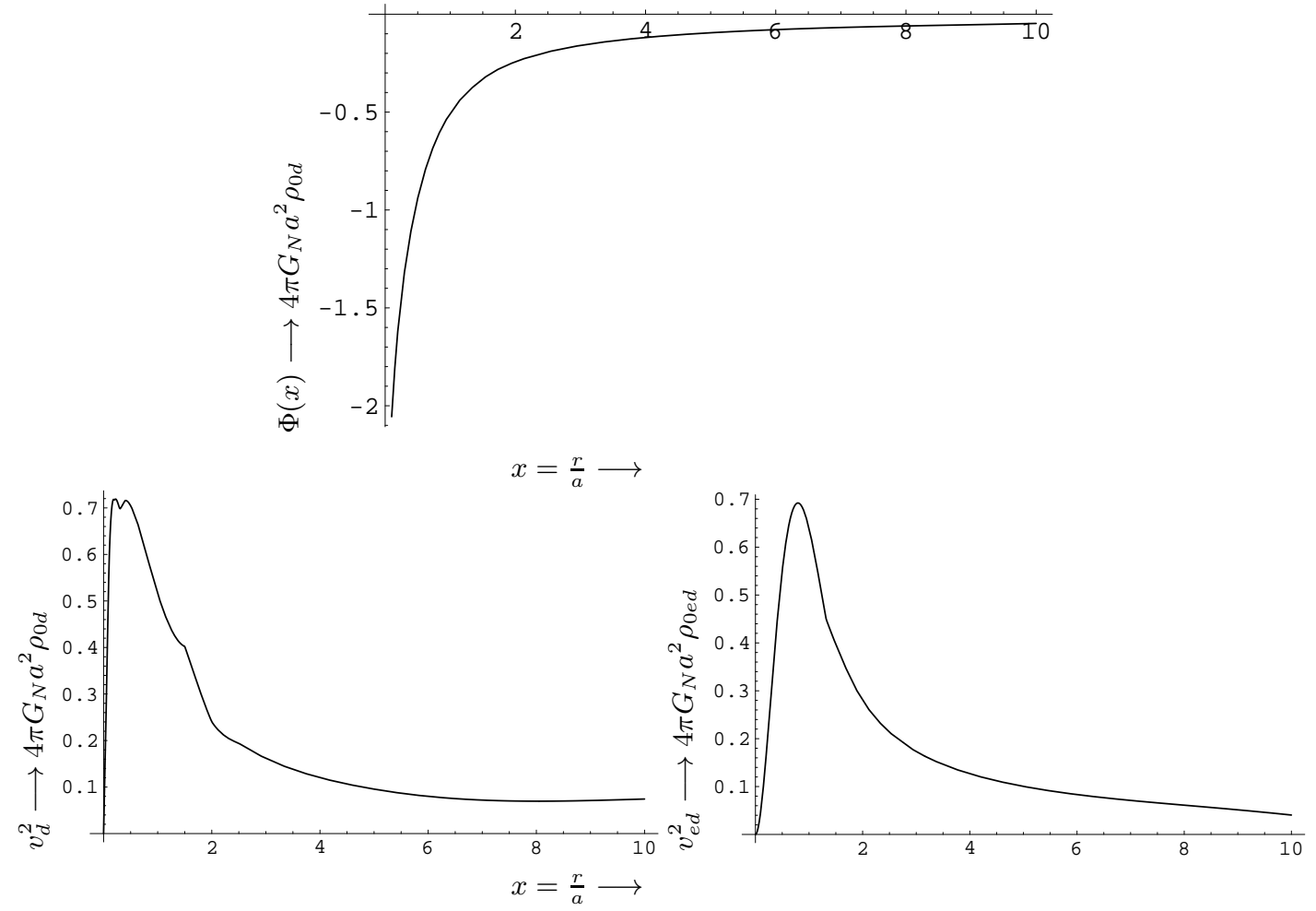

FIG. 3: On top we show the Potential in units of $4 \pi G_{N} a^{2} \rho_{0 d}$ on the plane of the galactic disc resulting from the ordinary matter distribution on the disc discussed in the text. At the bottom we show on the left the square of the rotational velocity resulting from the above density and on the right the same quantity resulting from an exponential density profile. 


\section{Dark matter density}

There are many halo density profiles, which have been employed. In the present case we will consider only spherical distributions, since it is not easy to extend the Eddington approach to deal with the most general case. Among the most commonly used are:

- The simple density profile

$$
\rho(x)=\frac{\rho_{0}}{1+x^{2}}, \quad x=\frac{r}{a}
$$

with $a$ the radius of the Galaxy. This profile has the advantage that the rotational velocity remains constant with the distance from the center of the galaxy becomes very large.

- Another simple profile is:

$$
\rho(x)=\frac{\rho_{0}}{x(1+x)^{2}}, \quad x=\frac{r}{a}
$$

suggested by N-body simulations 30 . This profile provides a better description of the expected density near the center of the galaxy. It does not, however, predict the constancy of the rotational velocities at large distances.

In the present work we will consider the density profile of Eq. (28)

Unfortunately with this density the potential diverges at infinity. On the other hand the solution to Poisson's equation is finite at the origin and it can be chosen to vanish there, i.e. it takes the form:

$$
\frac{\Phi}{\Phi_{0}}=\frac{\tan ^{-1}(x)}{x}+\frac{1}{2} \ln \left(1+x^{2}\right)-1
$$

One may choose a radius $x=c$ outside of which the density can be chosen to go faster to zero. One convenient choice is:

$$
\rho(x)=c_{2} \frac{\rho_{0}}{\left(1+x^{2}\right)^{2}}+c_{3} \frac{\rho_{0}}{\left(1+x^{2}\right)^{3}}
$$

with the requirement that at $x=c$ the density is continuous with a continuous derivative. we thus find:

$$
\rho_{>}(x)=\rho_{0}\left[\frac{2\left(c^{2}+1\right)}{\left(x^{2}+1\right)^{2}}-\frac{\left(c^{2}+1\right)^{2}}{\left(x^{2}+1\right)^{3}}\right]
$$

The obtained density is shown in Fig. 4. The potential in the region $x \prec c$ is the same as before:

$$
\frac{\Phi_{<}(x)}{\Phi_{0}}=I_{<}(x)+c_{3}
$$

The solution in the outer region takes the form:

$$
\frac{\Phi_{>}(x)}{\Phi_{0}}=I_{<}(c)+I_{>}(x)-I_{>}(c)-c_{3}
$$

where the constant $c_{3}$ can be chosen to make the potential vanish at some point and

$$
\begin{gathered}
I_{<}=\frac{\tan ^{-1}(x)}{x}+\frac{1}{2} \log \left(x^{2}+1\right) \\
I_{>}=\frac{1}{8}\left(\frac{\left(c^{2}+1\right)^{2}}{x^{2}+1}+\frac{\left(c^{2}-7\right) \tan ^{-1}(x)\left(c^{2}+1\right)}{x}+\frac{\left(-c^{4}+6 c^{2}+15\right) \tan ^{-1}(c)-c\left(c^{2}+15\right)}{x}\right)
\end{gathered}
$$



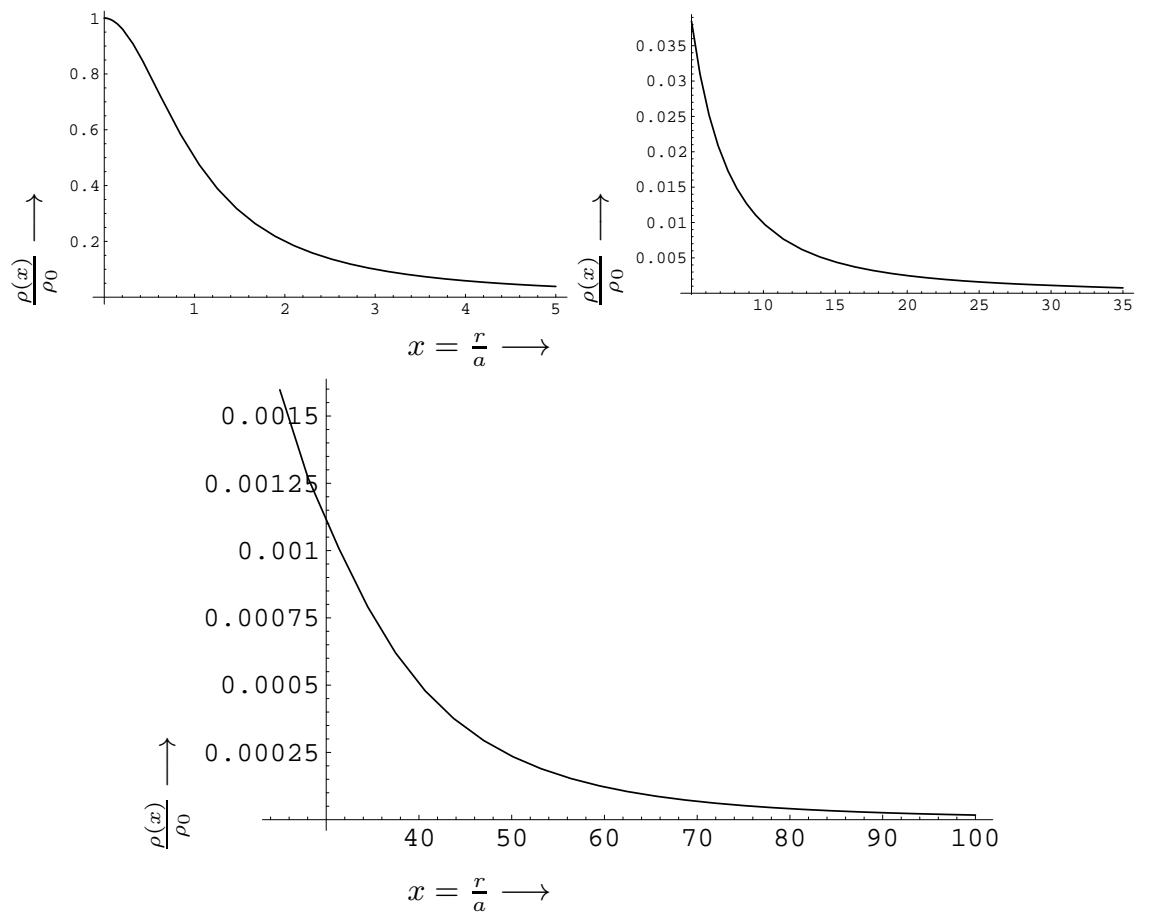

FIG. 4: The dark matter density distribution in dimensionless units.

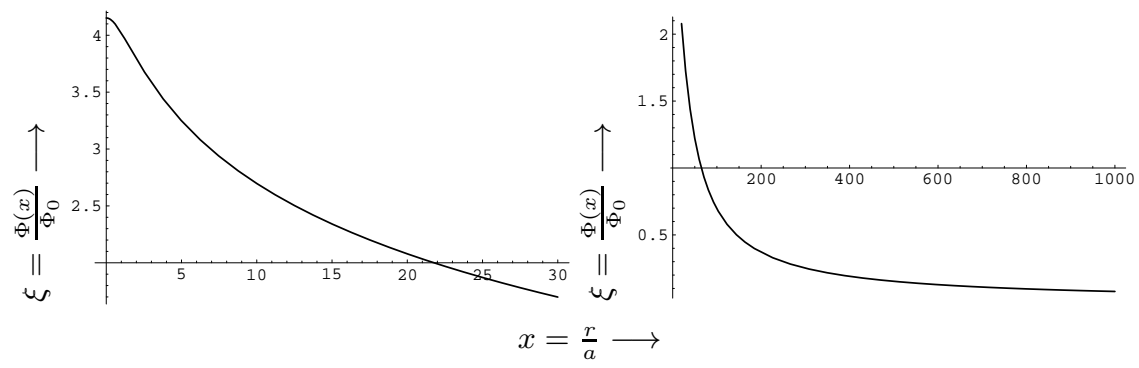

FIG. 5: The potential $\xi=\frac{\Phi(x)}{\Phi_{0}}$, 'i.e the absolute value of the potential in units $4 \pi G_{N} a^{2} \rho_{0}$, obtained with the density function shown in Fig. 4 and chosen to vanish at infinity. The potential drops to zero very slowly at large distances.

by choosing $c_{3}$ as

$$
c_{3}=\frac{1}{4}\left(2 \log \left(c^{2}+1\right)+7\right)
$$

the potential can be made to vanish at infinity. It is shown in Fig. [5 With the above ingredients it is not very difficult to obtain the function needed in the Eddington approach, namely $\eta(\xi)$. The results are shown in Fig. 6. In all cases Poisson's equation yields a relation between $\Phi_{0}$ and $\rho_{0}$, namely

$$
\Phi_{0}=-4 \pi G_{N} a^{2} \rho_{0}
$$

The obtained rotational velocity curve, due to dark mater alone, is given in Fig. 7 in units of $\sqrt{4 \pi G_{N} a^{2} \rho_{0}}$ From the observed rotational velocity one can fix the constant $\rho_{0}$. 


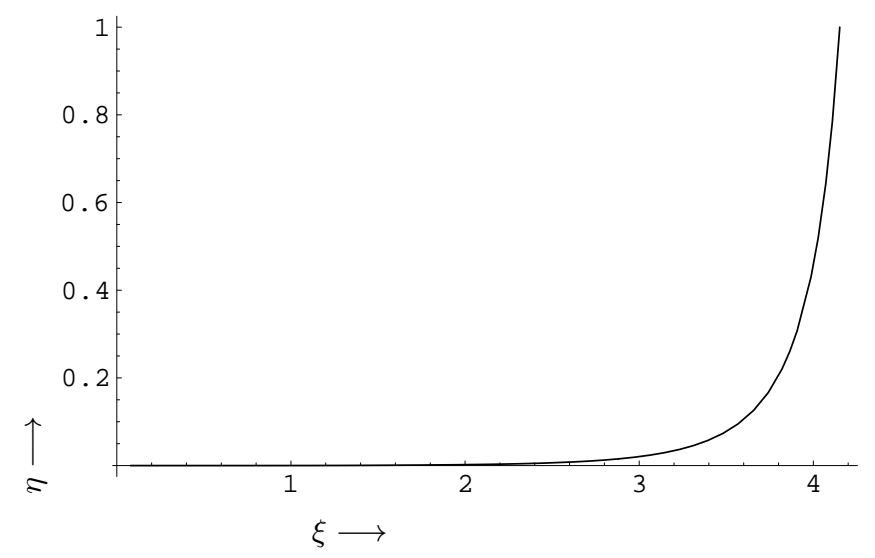

FIG. 6: The density $\eta$ as a function of the potential $\xi$

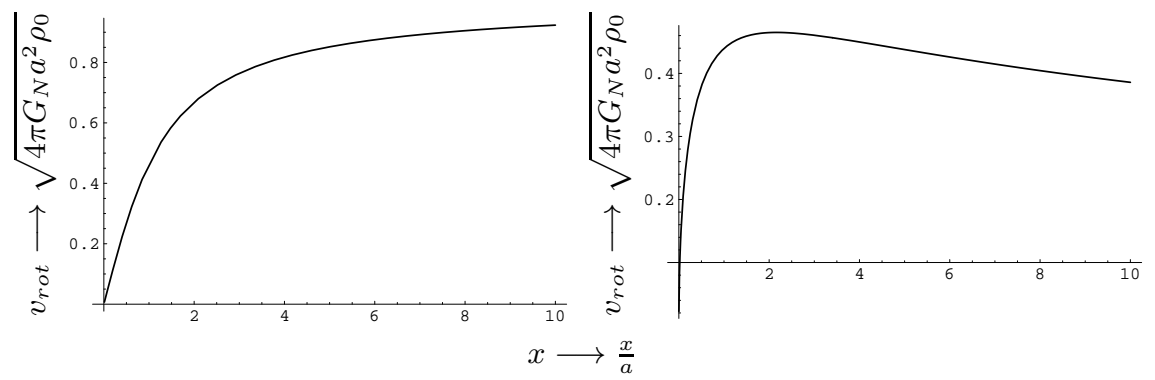

FIG. 7: The rotational velocity due to dark matter as a function of the distance in units of $\sqrt{4 \pi G_{N} a^{2} \rho_{0}}$. Shown on the left is the one obtained with the density profile of Eq. 28 adopted in this work, while on the right the NFW profile [30], see Eq. [29), was employed.

\section{Combining ordinary matter and dark matter}

We will now combine the three kinds of distribution considered above. We will assume that $\rho_{0 d}=\rho_{o b}=\rho_{s}$ and $\rho_{s}=\rho_{0}$. This means that in our vicinity the density of dark matter is equal to that of ordinary matter. Furthermore the ordinary matter density in our vicinity is equally split between the spherical and disc geometries. The obtained results are shown in Fig. 88 We see that

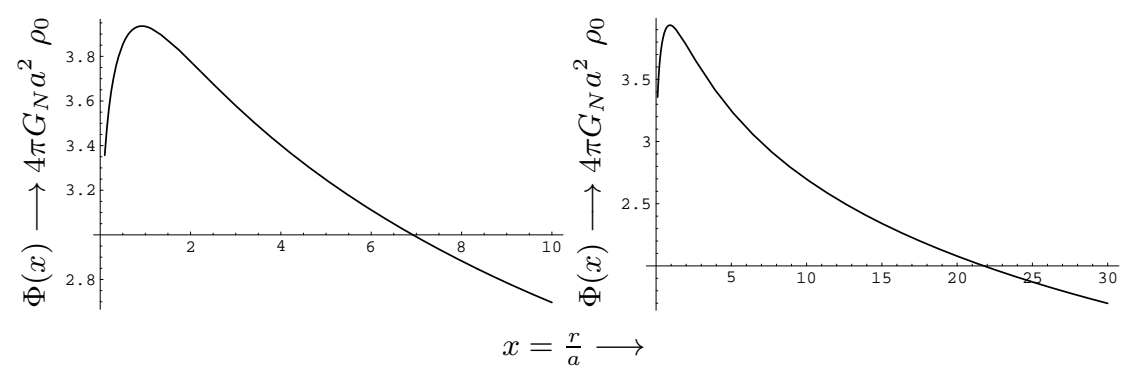

FIG. 8: The Potential due to all three matter distributions considered in the text.

in order to fit the rotational velocity of the sun, $220 \mathrm{~km} / \mathrm{s}$ we need a density $\rho_{0}=1.0 \times 10^{-21} \mathrm{~kg} / \mathrm{m}^{3}$. This is in agreement with the value of $0.3 \mathrm{GeV} / \mathrm{cm}^{3}=0.5 \times 10^{-21} \mathrm{~kg} / \mathrm{m}^{3}$ used in calculations of the rates for direct dark matter searches (in our model the dark component is half of the total). 


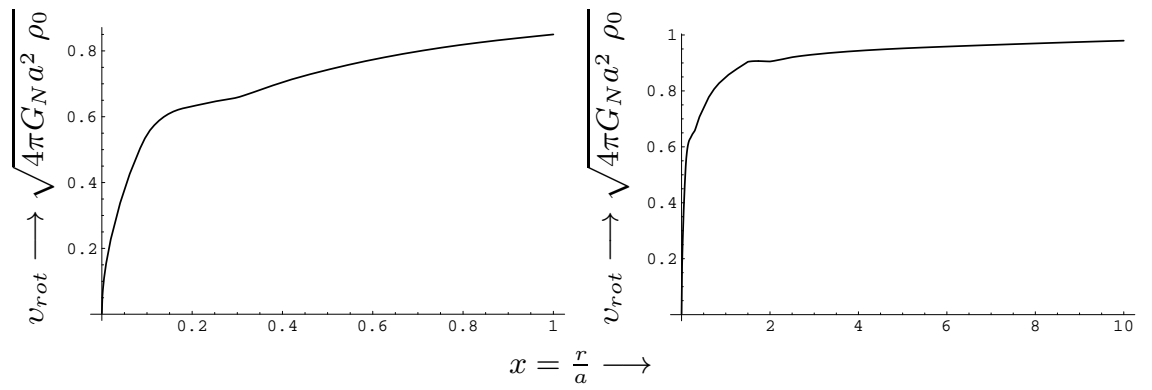

FIG. 9: The rotational velocity resulting from the potential of Fig. 8

\section{THE VELOCITY DISTRIBUTION IN OUR VICINITY}

It is clear that the velocity distribution and, in particular the maximum allowed velocity, is related to the escape velocity via the density $\rho_{0}$. For the moment we will ignore the asymmetric term and set $\alpha_{s}=0$.

\section{A. Dark Matter only}

The relation between the density and the potential has already been shown in Fig. 6 In this case we obtain the velocity distribution shown in Fig. 10] with the usual normalization imposed

$$
\int_{0}^{y_{m}} y^{2} f_{v}(y) d y=1
$$

Instead of the velocity we have used the dimensionless quantity y:

$$
y=\frac{v}{\sqrt{4 \pi G_{N} a^{2} \rho_{0}}} .
$$

For comparison we present the velocity distributions obtained with the profile of Eq. (29) in Fig.

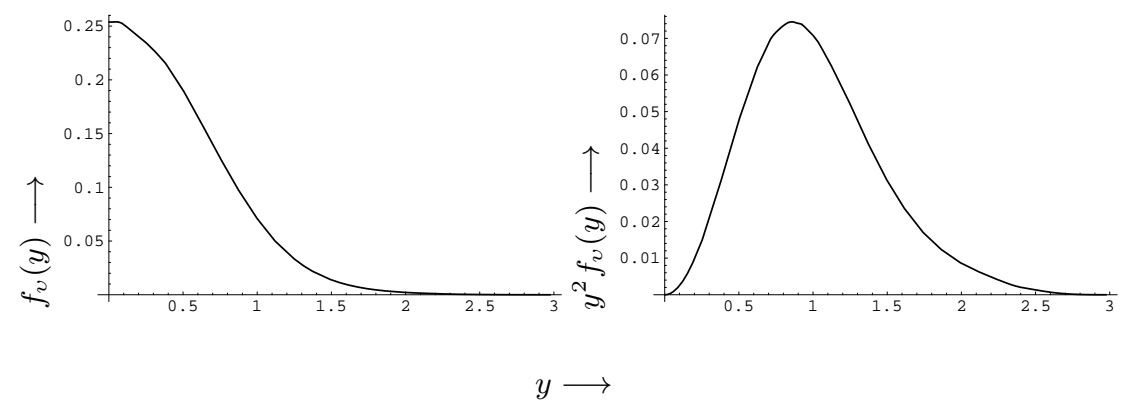

FIG. 10: The velocity distributions $f_{v}(y)$ and $y^{2} f_{v}(y)$ in units of the parameter $\sqrt{4 \pi G_{N} a^{2} \rho_{0}}$, i.e. $y=$ $v / \sqrt{4 \pi G_{N} a^{2} \rho_{0}}$ obtained with the density profile of Eq. (28)

11 and with the standard M-B distribution in Fig. 12 The maximum velocity allowed by our distribution is only $y_{m}=2.8$. Assuming $a=3.1 \times 10^{20} \mathrm{~m}$ and $\left.\rho\right|_{x=1}=\rho_{s} / 2$, i.e. half of the local density to be due to dark matter, we find $\rho_{0}=\rho_{s}$ which yields $v_{m}=y_{m} \sqrt{4 \pi G_{N} a^{2} \rho_{0}}=$ $2.8 \times 270 \mathrm{~km} / \mathrm{s}=7.5 \times 10^{5} \mathrm{~m} / \mathrm{s}$. This is a bit higher than the escape velocity, $v_{e s c}=6.2 \times 10^{5} \mathrm{~m} / \mathrm{s}$, assumed in theories employing the M-B distribution. For comparison we present the same quantities for the M-B distribution in Figs 12 12 Since the M-B distribution does not go to zero at finite values 


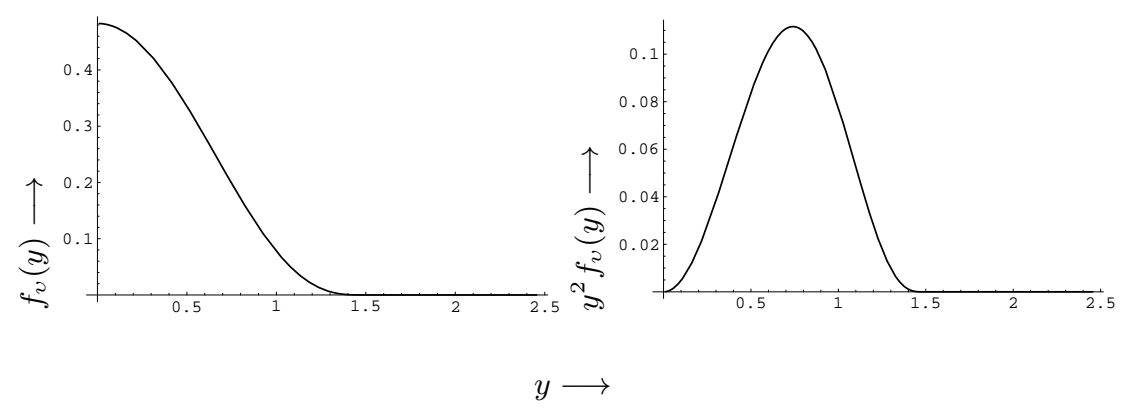

FIG. 11: The velocity distributions $f_{v}(y)$ and $y^{2} f_{v}(y)$ in units of the parameter $\sqrt{4 \pi G_{N} a^{2} \rho_{0}}$, i.e. $y=$ $v / \sqrt{4 \pi G_{N} a^{2} \rho_{0}}$, obtained with the density profile of Eq. 29]

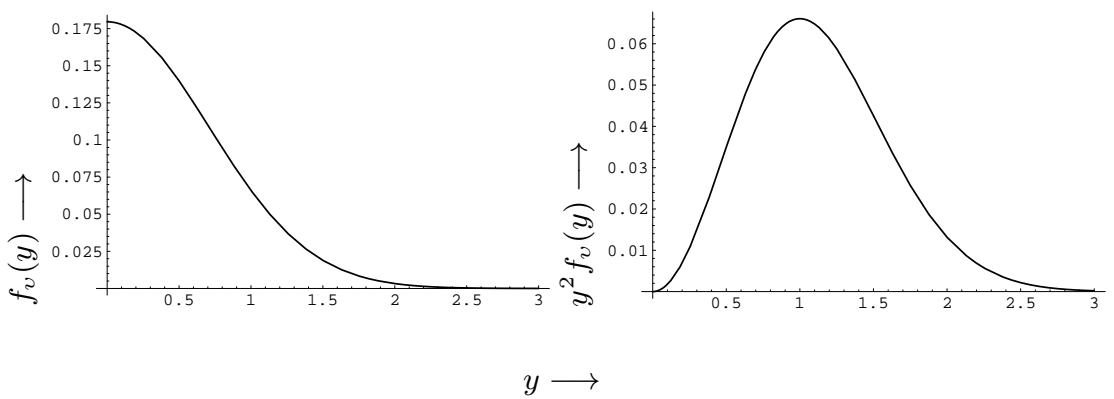

FIG. 12: The same as in Fig. 10] in the case of the M-B distribution. Now, however, the parameter $y$ is the velocity in units of the sun's rotational velocity, i.e. $y=v / v_{0}$ with $v_{0}=2.2 \times 10^{5} \mathrm{~m} / \mathrm{s}$. Note that, in these units, the escape velocity is at 2.84

of the velocity, the maximum allowed velocity is set by hand equal to the escape velocity.

We should emphasize that in this approach we encounter two characteristic velocities. One is the rotational velocity and the other the maximum allowed velocity $v_{m}$. The first depends on the square root of the derivative of the potential, while the second scales with the square root of the potential itself.

\section{B. Both ordinary matter and dark matter}

To improve the situation one will attempt to include gravity. Since it is very hard to incorporate the disc geometry into the Eddington approach, we will attempt to mimic the gravitational effects of ordinary matter with a spherical distribution like the one discussed above, but twice a large, so that in our position the usual and cold dark matter contribution are about equal. The thus obtained function $\eta=\eta(\xi)$ is shown in Fig. 13 .

In this case we find $y_{m}=3.2$. In other words the effect of ordinary matter is small, since the dark matter potential in our vicinity is about 12 times stronger than the potential due to ordinary matter. Again the condition $\left.\rho\right|_{x=1}=\rho_{s}$ implies that overall constant $\rho_{0}$ in the density distribution is $\rho_{s}$, which gives $v_{m}=3.1 \times 2.7 \times 10^{5} \mathrm{~m} / \mathrm{s}=8.0 \times 10^{5} \mathrm{~m} / \mathrm{s}$. With this modification the obtained results for the velocity distribution are shown in Figs 14 We see that the inclusion of gravity has very little effect on the velocity distribution. So in what follows we will consider only the dark matter component. 


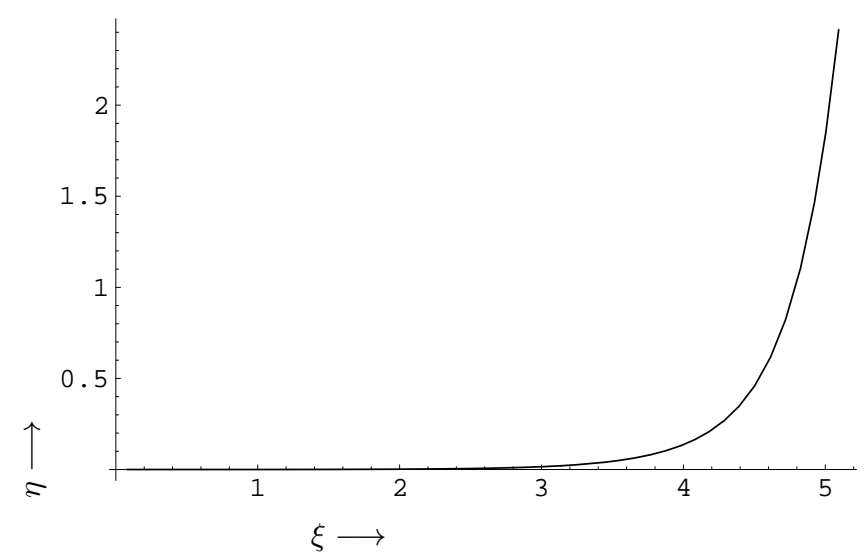

FIG. 13: The density $\eta$ as a function of the potential $\xi$, when both ordinary and dark matter are included.

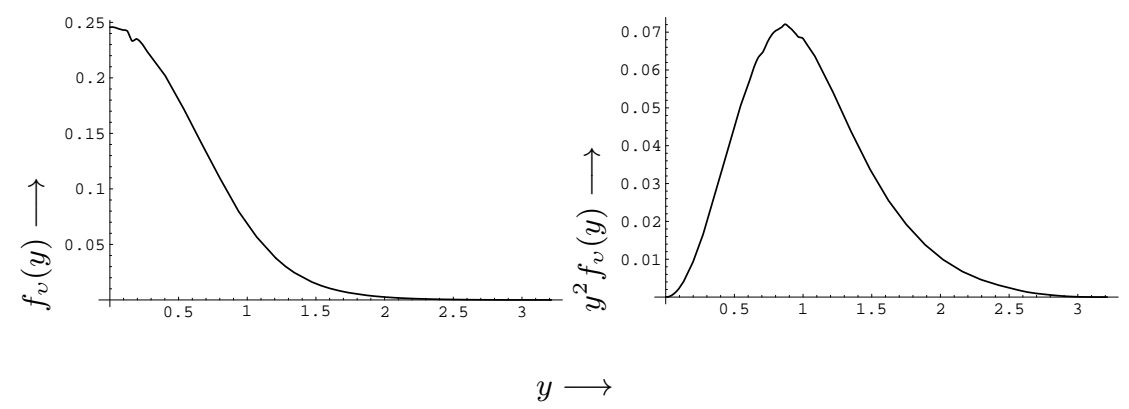

FIG. 14: The same as in Fig. 10] when both ordinary and dark matter are included.

\section{DIRECT DARK MATTER RATES}

In the present work we find it convenient to write the event rate in the form ${ }^{1}$ :

$$
R=\bar{K}\left[c_{c o h}\left(A, \mu_{r}(A)\right) \sigma_{p, \chi^{0}}^{S}+c_{s p i n}\left(A, \mu_{r}(A)\right) \sigma_{p, \chi^{0}}^{s p i n} \zeta_{s p i n}\right]
$$

In this expression $\sigma_{N, \chi^{0}}^{S}$ is the LSP-nucleon scalar cross section, while $\sigma_{p, \chi^{0}}^{s p i n}$ is the proton cross section associated with the spin. The quantity $\zeta_{\text {spin }}$ is given by [15]:

$$
\begin{gathered}
\zeta_{\text {spin }}=\frac{1}{3\left(1+\frac{f_{A}^{0}}{f_{A}^{1}}\right)^{2}} S(u) \\
\left.S(u) \approx S(0)=\left[\left(\frac{f_{A}^{0}}{f_{A}^{1}} \Omega_{0}(0)\right)^{2}+2 \frac{f_{A}^{0}}{f_{A}^{1}} \Omega_{0}(0) \Omega_{1}(0)+\Omega_{1}(0)\right)^{2}\right]
\end{gathered}
$$

The static spin matrix elements are obtained in the context of a given nuclear model (see, e.g., previous work [17]- 18], 15, 40] and references therein). Even, though most of what we are going to say applies in the case of the spin induced rate we are not going to further elaborate here.

${ }^{1}$ see our previous recent work [15, 40] for a more detailed discussion and additional references 
In Eq. (37) $\bar{K}$ is given by:

$$
\bar{K}=\frac{\rho(0)}{100 \mathrm{GeV}} \frac{m}{m_{p}} \sqrt{\left\langle v^{2}\right\rangle} \simeq 16010^{-4}(p b)^{-1} y^{-1} \frac{\rho(0)}{0.3 G e V c m^{-3}} \frac{m}{1 K g} \frac{\sqrt{\left\langle v^{2}\right\rangle}}{280 k m s^{-1}}
$$

and

$$
\begin{gathered}
c_{c o h}\left(A, \mu_{r}(A)\right)=\frac{100 \mathrm{GeV}}{m_{\chi^{0}}}\left[\frac{\mu_{r}(A)}{\mu_{r}(p)}\right]^{2} A t_{c o h}(A) \\
c_{\text {spin }}\left(A, \mu_{r}(A)\right)=\frac{100 G e V}{m_{\chi^{0}}}\left[\frac{\mu_{r}(A)}{\mu_{r}(p)}\right]^{2} \frac{t_{s p i n}(A)}{A}
\end{gathered}
$$

The parameters $c_{c o h}\left(A, \mu_{r}(A)\right), c_{s p i n}\left(A, \mu_{r}(A)\right)$, which give the relative merit for the coherent and the spin contributions in the case of a nuclear target compared to those of the proton, have already been tabulated [40] for energy cutoff $Q_{\min }=0$ and $10 \mathrm{keV}$.

Via Eq. (37) we can extract the nucleon cross section from the data. The most interesting quantity is $t_{c o h}(A)$. It is defined as:

$$
t_{c o h}=\int_{u_{\min }}^{u_{\max }} \frac{d t_{c o h}}{d u} d u
$$

$\mathrm{u}$ is the energy transfer to the nucleus (in dimensionless units, see below)

$$
\begin{gathered}
u_{\min } \Leftrightarrow \text { detector threshold } \\
u_{\max } \Leftrightarrow \text { maximum WIMP velocity } \\
\frac{d t_{c o h}}{d u}=\sqrt{\frac{2}{3}} \frac{v_{p a r}}{v_{0}} T(u), T(u)=a^{2}|F(u)|^{2} \Psi(a \sqrt{u})
\end{gathered}
$$

for the coherent mode and

$$
\begin{gathered}
t_{\text {spin }}=\int_{u_{\text {min }}}^{u_{\max }} \frac{d t_{\text {spin }}}{d u} d u \\
\left.\frac{d t_{\text {spin }}}{d u}=\sqrt{\frac{2}{3}} \frac{v_{\text {par }}}{v_{0}} T(u), T(u)=a^{2} F_{11} u\right) \Psi(a \sqrt{u})
\end{gathered}
$$

where $v_{p a r}=v_{m}$ in the present approach and $v_{p a r}=v_{0}$ in the case of the M-B distribution and The nucleon cross sections, which carry the dependence on the particle model parameters, are the most important ones, but they are not of interest in our present calculation. One such parameter is, of course, the WIMP mass. In the above expressions $F(u)$ is the form factor, entering the coherent scattering and $F_{11}(u)$ is the spin response function entering via the axial current. The function $\Psi$ depends on the WIMP distribution velocity employed and is a function of the energy $Q$ transferred to the nucleus

$$
u=\frac{Q}{Q_{0}} \quad, \quad Q_{0}=\frac{4 A m_{p}}{b^{2}}=4.1 \times 10^{4} A^{-4 / 3} \mathrm{KeV}
$$

where $A$ is the nuclear mass number and the dimensionless parameter $a$ is given by:

$$
a=\left[\sqrt{2} \mu_{r} b v_{p a r}\right]^{-1},
$$


where $\mu_{r}$ is the reduced mass of the WIMP-nucleus system and $b$ is the (harmonic oscillator) size parameter.

The function, which is basic to us, $\Psi$, is given by

$$
\Psi(a \sqrt{u})=\int_{a \sqrt{u}}^{y_{m}} d y \int_{0}^{\pi} \sin \theta d \theta \int_{0}^{2 \pi} d \phi y f_{v}(y, \theta, \phi)
$$

with

$$
f_{v}(y, \theta, \phi)=f_{v}\left(\sqrt{y^{2}+2 y y_{\text {sun }} \cos \theta+y_{\text {sun }}^{2}}\right)
$$

where $\theta$ is the polar angle as measured from the direction of the sun's motion and $y_{\text {sun }}$ is the sun's velocity in units of $v_{m}$. Note that since the argument of the function $f_{v}$ is constrained to be less than $y_{m}$. If $y_{m}$ is small the allowed region in the $(y, \xi)$ space is very restricted. The function $\Psi(a \sqrt{u})$ is plotted in Fig. 15] The function $\Psi$ for both distributions is shown in Fig. 15. We should stress

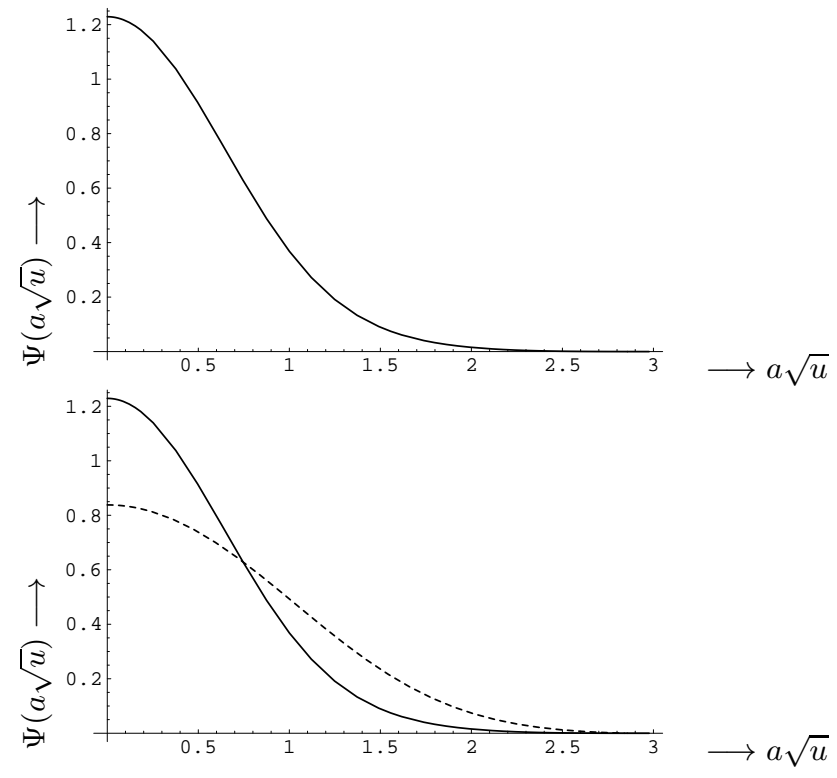

FIG. 15: On the top we show the function $\Psi(a \sqrt{u})$ which enters the differential (with respect to the energy transfer $\mathrm{u}$ ) event rate in dark matter searches. It has been obtained in the context of the Eddington approach as discussed in the text. For comparison we present at the bottom the same function (continuous curve) together with that obtained using the M-B distribution (dotted curve).

that the relative differential rate $\frac{1}{R} \frac{d R}{d u}$ can be obtained by combining the above results of $\Psi(a \sqrt{u}$ with the nuclear form factor for the target of interest. The dependence on the WIMP mass comes through the parameter $a$.

\section{APPLICATION IN THE CASE OF THE TARGET ${ }^{127}$ I}

In this section we are going to apply the above formalism in the case of a popular target, ${ }^{127} \mathrm{I}$, which is an odd mass target and can detect both the coherent and the spin modes of the WIMPnuclear interaction. We will include in our only the coherent mode but we do not expect any real differences as far as the quantity $\frac{1}{R} \frac{d R}{d u}$ is concerned. The nuclear form factor employed was obtained in the shell model description of the target and is shown in Fig. 16. The quantity $\frac{d t_{c o h}}{d u}$ obtained both for our distribution as well as for the familiar M-B distribution is shown in Figs 17,20 for various WIMP masses $m_{\chi}$. It is clear that the differential rate is a fast decreasing function of the energy transfer. This is particularly true for low WIMP masses. 


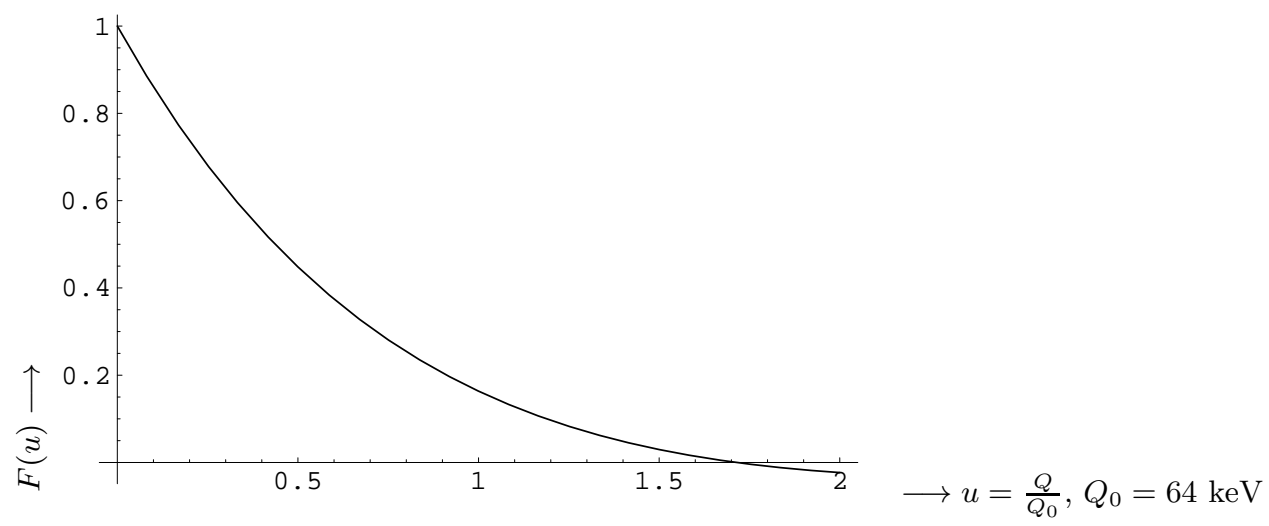

FIG. 16: The form factor employed in our calculation. $u$ is the energy transfer to the nucleus in units of $Q_{0}$, i.e. $u=Q / Q_{0}$, with $Q_{0}=64 \mathrm{keV}$.
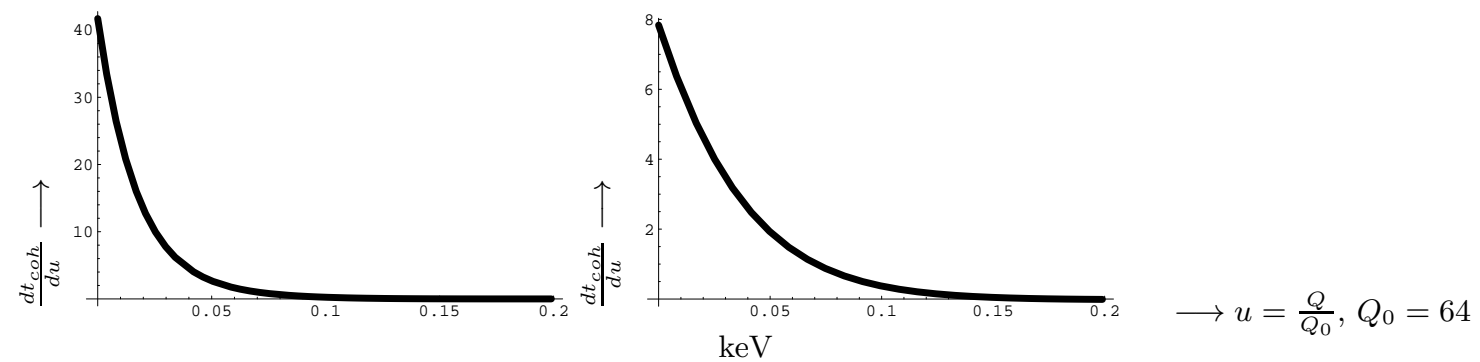

FIG. 17: On the left we show the quantity $\frac{d t_{c o h}}{d u}$ for $m_{\chi}=10 \mathrm{GeV}$ in the case of the distribution obtained in this work. On the right we show the same quantity in the case of the M-B distribution. The expression for $t_{\text {spin }}$ is similar. Note that for such a small WIMP mass the differential rate drops very fast as a function of the energy transfer.
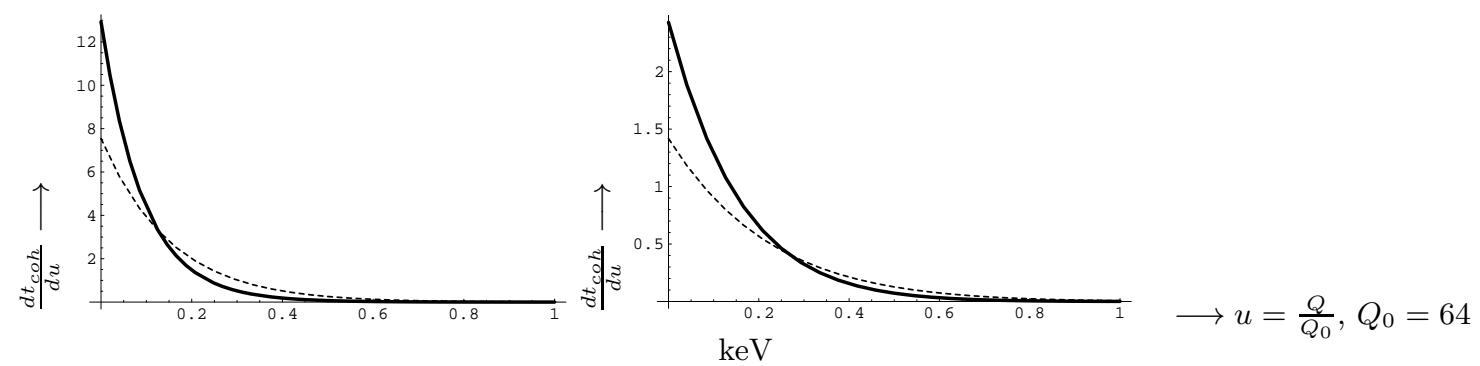

FIG. 18: The same as in Fig. [17 for $m_{\chi}=30 \mathrm{GeV}$ (solid line) and $m_{\chi}=50 \mathrm{GeV}$ (dashed line).

Integrating the differential rate from zero to $u_{\max }=y_{\max }^{2} / a^{2}$, with $y_{\max }=y_{m}\left(y_{\max =2.84}\right)$ for the present (M-B) distributions respectively, we find the total rate $\frac{R}{R}$ as a function of the WIMP mass. The results are shown in Fig. 21] As we have already mentioned the nucleon cross sections also depend on the LSP mass. So the absolute event rates, which include these cross sections, are expected to drop even faster as a function of the LSP mass. In practice, however, the detectors have a low energy threshold. So only the event rates above an energy transfer $Q_{t h}$ can be detected. Thus we present the relative total rates $t_{c o h}$ as a function of $Q_{t h}$ in figs 2224 From these plots we see that it is crucial for experiments to lower the threshold energy as much as possible. This is particularly true for small WIMP masses. 


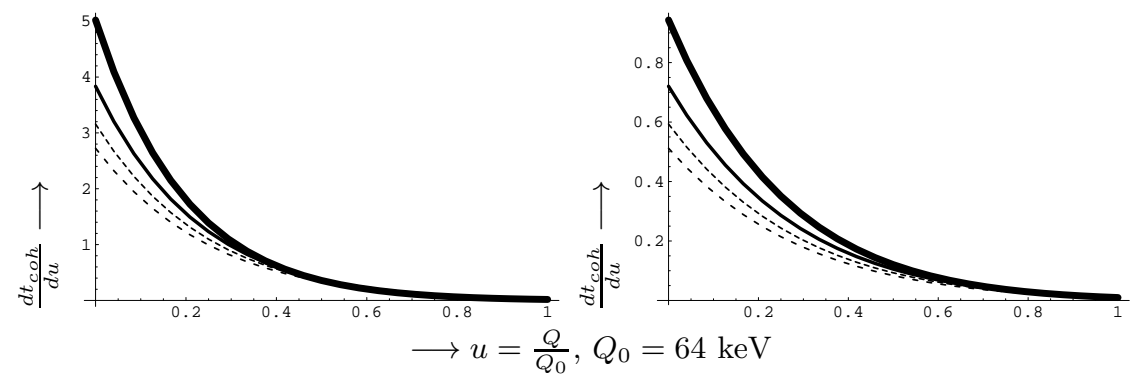

FIG. 19: The same as in Fig. 17 for $m_{\chi}=75 \mathrm{GeV}$ (thick solid line), $m_{\chi}=100 \mathrm{GeV}$ (thin solid line), $m_{\chi}=125 \mathrm{GeV}$ (short dashed line) and $m_{\chi}=150 \mathrm{GeV}$ (long dashed line).
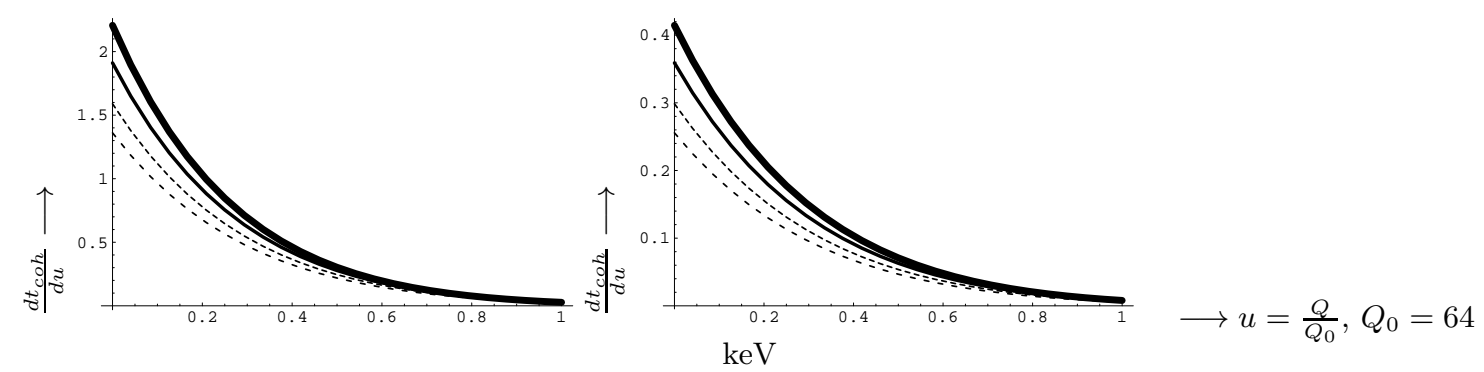

FIG. 20: The same as in Fig. 19] for $m_{\chi}=200 \mathrm{GeV}$ (thick solid line), $m_{\chi}=250 \mathrm{GeV}$ (thin solid line), $m_{\chi}=350 \mathrm{GeV}$ (short dashed line) and $m_{\chi}=500 \mathrm{GeV}$ (long dashed line).
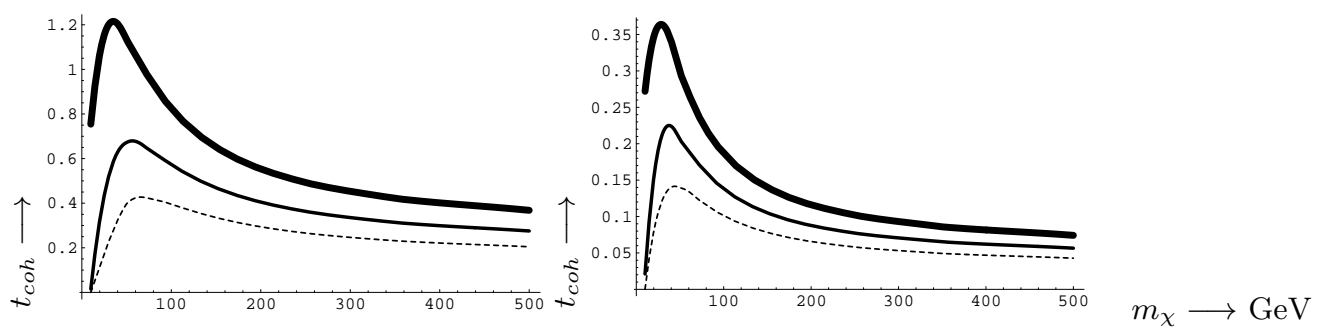

FIG. 21: On the left we show the quantity $t_{c o h}$ in the case of the present distribution as a function of the WIMP mass and threshold energy $\left(Q_{t h}=0 \Longleftrightarrow\right.$ thick solid curve, $Q_{t h}=5 \mathrm{keV} \Longleftrightarrow$ fine solid curve and $Q_{t h}=10 \mathrm{keV} \Longleftrightarrow$ dashed curve). On the right we show the same quantity in the case of the M-B. It is clear that the rates decrease as the threshold energy increases. This is is especially true for low LSP mass
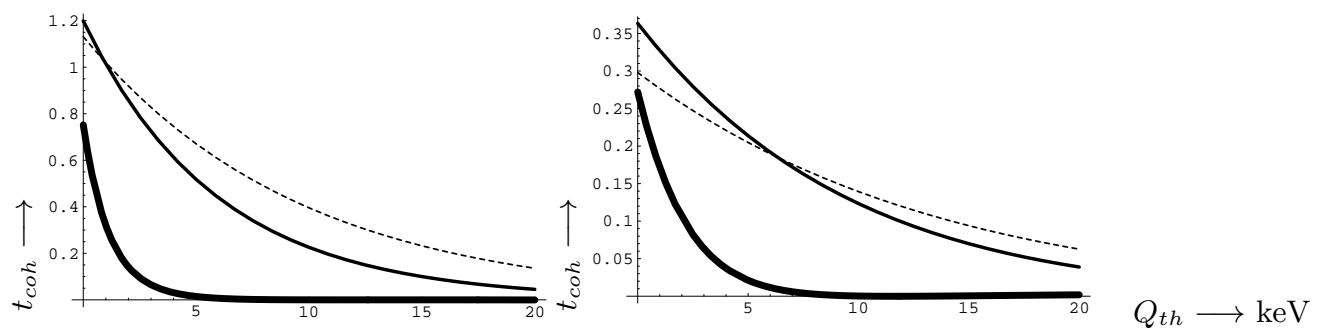

FIG. 22: The relative rates $t_{c o h}$ as a function of threshold energy for WIMP masses $m_{\chi}=10$ (thick solid line), $m_{\chi}=30 \mathrm{GeV}$ (fine solid line) and $m_{\chi}=50 \mathrm{GeV}$ (dashed line). The results on the left correspond to the present distribution, while those on the right to the M-B distribution. 


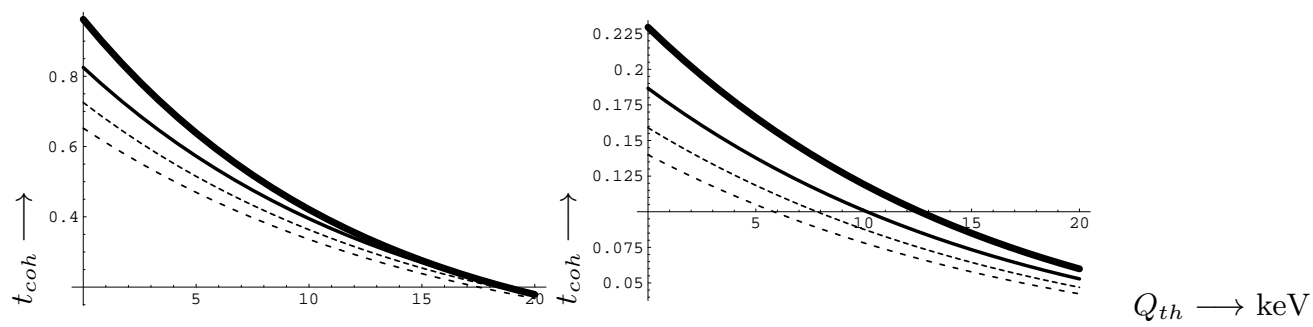

FIG. 23: The same as in Fig. 22 for $m_{\chi}=75 \mathrm{GeV}$ (thick solid line), $m_{\chi}=100 \mathrm{GeV}$ (thin solid line), $m_{\chi}=125 \mathrm{GeV}$ (short dashed line) and $m_{\chi}=150 \mathrm{GeV}$ (long dashed line).
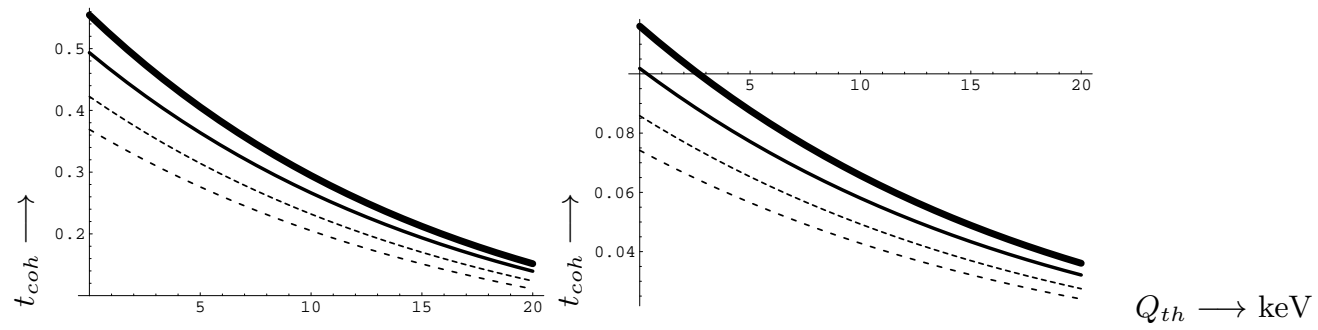

FIG. 24: The same as in Fig. 23] for $m_{\chi}=200 \mathrm{GeV}$ (thick solid line), $m_{\chi}=250 \mathrm{GeV}$ (thin solid line), $m_{\chi}=350 \mathrm{GeV}$ (short dashed line) and $m_{\chi}=500 \mathrm{GeV}$ (long dashed line).

\section{MODULATION}

In the above discussion we did not take into account the motion of the Earth. The expected event rates, however, are very small due to the smallness of the nucleon cross sections not discussed in this work. So the experiments must fight against formidable backgrounds. Fortunately there are some signatures of the WIMP-nuclear interaction, which must be exploited. One such comes from the fact that the event rates depend on the relative velocity between the WIMP and the target. The most important velocity dependent contribution comes from the rotation of the Earth around the sun with a velocity $v_{1}=0.27 v_{0}$. It turns out that only the component of the Earth's velocity along the sun's direction of motion, $\left(v_{1}\right)_{z}=0.135 \cos \alpha$, is relevant ( $\alpha$ is the phase of the Earth, $\alpha=0$ around June 3nd). We can thus apply the above formalism by

$$
y_{\text {sun }} \Rightarrow y_{\text {sun }}(1+0.068 \cos \alpha)
$$

Thus Eqs 44 and 46 become

$$
T(u) \Longrightarrow a^{2}|F(u)|^{2}[\Psi(a \sqrt{u})+H(a \sqrt{u}) \cos \alpha]
$$

for the coherent mode and similarly for the spin We will examine the modulation effect in the case of ${ }^{127} \mathrm{I}$. The behavior of the function $H(a \sqrt{u})$ is exhibited in Fig. 25] We notice the sign change of $H$ as the energy transfer changes. Integrating the differential rates we obtain the total rates, which now now take the form:

$$
t \Longrightarrow t(1+h \cos \alpha)
$$

of course, on the parameter $a$. Quite generally for a light nuclear system and relatively heavy WIMP, the parameter $a$ is large, i.e. the lower part of the Fig The exact behavior of the modulation of event rates depends, of course, on the parameter $a$ and the nuclear form factor. Quite generally for light nuclear targets, i.e. large $a$, the lower range of Fig. 25] does not enter and the modulation $h$ is not suppressed. On the other hand, for intermediate and heavy nuclei, the lower part tends to cancel the effect of the upper part of Fig. 25] This leads to a reduction of the modulation of the total rates 

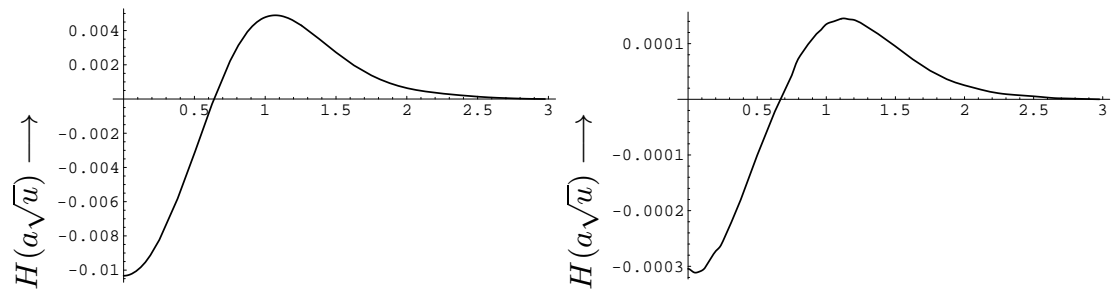

$$
\longrightarrow a \sqrt{u}
$$

FIG. 25: On the left we show the function $H(a \sqrt{u})$ governing the differential modulation amplitude as a function the energy transfer $u$. On the right we show the unimportant higher order effects $\sim \cos 2 \alpha$. We note the sign change as the energy transfer increases.

and even in a change of sign of $h$, i.e. minimum in June and maximum in December. The behavior of the quantity $h$, in the case of the present velocity distribution foe ${ }^{127} I$, is exhibited in Fig. [26] We see that the modulation predicted by the present distribution is much smaller than that obtained with a M-B distribution (see Fig. 27). This is true regardless of the energy cut off. Similar results have been obtained in a modified M.B. distribution [41] obtained in a model that couples gravity to a scalar field. This way the characteristic velocity is not $v_{0}$, the sun's rotational velocity, but $\prec v_{d} \succ \sqrt{2} \gg v_{0}$. We should emphasize that $h$ is the ratio of the modulated rate to the average

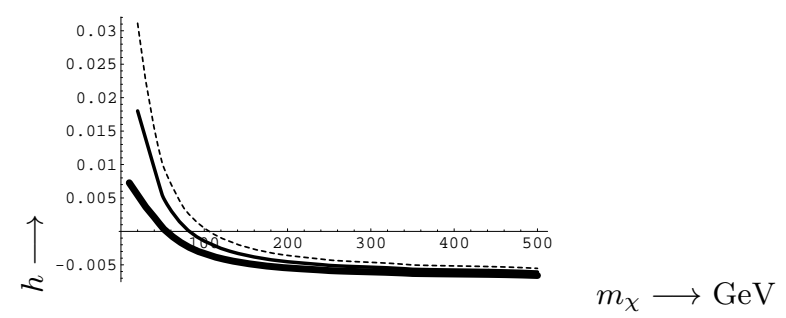

FIG. 26: The modulation function $h$ is shown as a function of the WIMP mass for various threshold energies obtained with the present velocity distribution. In this figure: $Q_{t h}=0 \Longleftrightarrow$ thick solid curve, $Q_{t h}=5 \mathrm{keV}$ $\Longleftrightarrow$ fine solid curve and $Q_{t h}=10 \mathrm{keV} \Longleftrightarrow$ dashed curve.

rate. In other words the increase of $h$ as the threshold energy increases, results from the fact that the average rate (denominator) decreases much faster than the modulated rate (numerator). So the increase in $h$, which is a good signal against background, is not cheap. It comes at the expense of the number of counts.
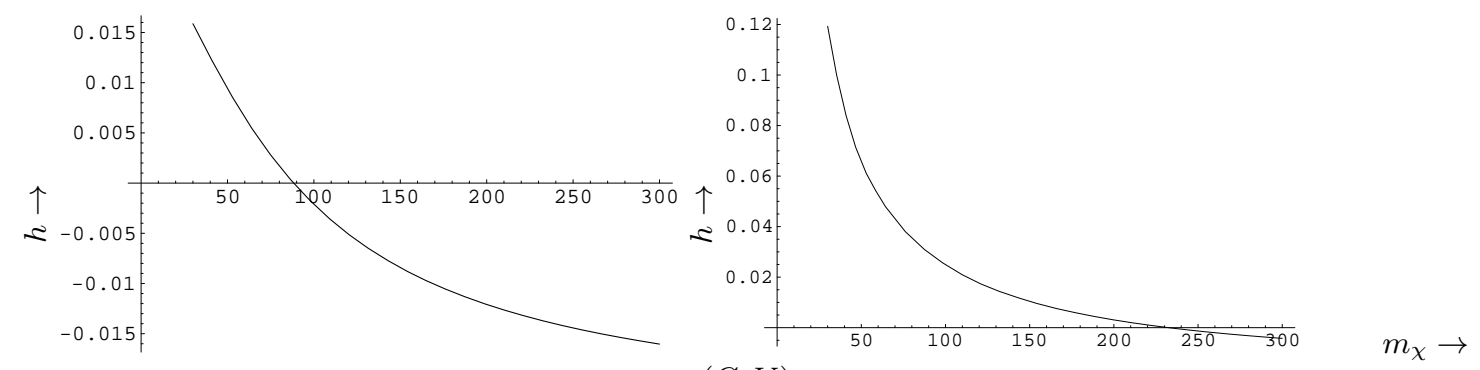

$(\mathrm{GeV})$

FIG. 27: The modulation amplitude $h$ as a function of the WIMP mass obtained with a M-B distribution in the case of ${ }^{127} \mathrm{I}$ for $Q_{\text {min }}=0$ on the left and $Q_{m i n}=10 \mathrm{keV}$ on the right. For the definitions see text. 


\section{CONCLUSIONS}

In the present paper we discussed the dependence of the direct dark matter event rates on the density and velocity distribution of WIMP's. This was done in a self consistent way by applying Eddington's approach. Even though at present only spherically symmetric matter distributions can be treated this way, one can draw the following conclusions:

- Simple dark matter distributions can adequately describe the rotational curves of both spherical and disc galaxies. With the assumed form of the density its scale was determined by fitting the rotational velocity of the sun. The value of dark matter density extracted is in agreement with that hitherto employed in dark matter calculations, $\simeq 0.3 \mathrm{GeV} / \mathrm{cm}^{3}$.

- From the assumed density profile we obtained the distribution function. Evaluation of the distribution function in our vicinity yields the velocity distribution. This velocity distribution automatically vanishes for velocities larger than a velocity $v_{m} \cdot v_{m}$ depends on the square root of the potential in our vicinity. It is quite different from the rotational velocity, which depends on the square root of the derivative of the potential.

- The velocity distribution obtained is not very different from the standard M-B in the range of velocities of interest to dark matter searches.

- We focused our attention on the factor $t$ entering dark matter rates, which is independent of the nucleon cross section, i.e. it holds for all heavy WIMPs. The values obtained in our model are larger than those obtained using the M-B distribution. The relative magnitude depends on the WIMP mass. For small WIMP masses the obtained results are 3-4 times larger than those of the M-B case.

- We also computed the modulation amplitudes $H(u)$ and $h$. We find that both are more suppressed than those obtained with the M-B distribution. It seems that the precise value of the modulation sensitively depend on the assumed velocity distribution (the form of the function $H(u)$ is independent of the assumed nuclear form factor). It appears, though, that the modulation obtained with the present velocity distribution is perhaps too small to be of practical interest experimentally.

We did not consider in this work asymmetric velocity distributions. Another signature not discussed in this work is the asymmetry, with respect to the sun's direction of motion, expected in directional experiments [2]. In such experiments, which detect not only the energy of the recoiling nucleus, but its direction of motion as well, the modulation is expected to be direction dependent. It is expected to be quite large in some directions. Such effects in the context of the present approach are currently under study.

\section{Acknowledgements}

This work was supported by the European Union under the contract MRTN-CT-2004-503369 as well as the program PYTHAGORAS-1. The latter is part of the Operational Program for Education and Initial Vocational Training of the Hellenic Ministry of Education under the 3rd Community Support Framework and the European Social Fund.

[1] S. Hanary et al: Astrophys. J. 545, L5 (2000); J.H.P Wu et al: Phys. Rev. Lett. 87, 251303 (2001); M.G. Santos et al: Phys. Rev. Lett. 88, 241302 (2002).

[2] P. D. Mauskopf et al: Astrophys. J. 536, L59 (2002);

S. Mosi et al: Prog. Nuc.Part. Phys. 48, 243 (2002);

S. B. Ruhl al, astro-ph/0212229 and references therein. 
[3] N. W. Halverson et al: Astrophys. J. 568, 38 (2002)

L. S. Sievers et al: astro-ph/0205287 and references therein.

[4] G. F. Smoot et al (COBE Collaboration), Astrophys. J. 396, L1 (1992).

[5] A. H. Jaffe et al, Phys. Rev. Lett. 86, 3475 (2001).

[6] D. N. Spergel et al, Astrophys. J. Suppl. 148, 175 (2003).

[7] D. P. Bennett et al., Phys. Rev. Lett. 74, 2867 (1995).

[8] M. W. Goodman and E. Witten, Phys. Rev. D 31, 3059 (1985).

[9] J. Ellis and L. Roszkowski, Phys. Lett. B 283, 252 (1992).

[10] A. Bottino et al., Phys. Lett B 402, 113 (1997).

R. Arnowitt. and P. Nath, Phys. Rev. Lett. 74, 4592 (1995); Phys. Rev. D 54, 2374 (1996); hep-ph/9902237

V. A. Bednyakov, H.V. Klapdor-Kleingrothaus and S.G. Kovalenko, Phys. Lett. B 329, 5 (1994).

[11] T. S. Kosmas and J. D. Vergados, Phys. Rev. D 55, 1752 (1997).

[12] J. D. Vergados, J. of Phys. G 22, 253 (1996).

[13] M. Drees and M. M. Nojiri, Phys. Rev. D 48, 3843 (1993); Phys. Rev. D 47, 4226 (1993).

[14] T. P. Cheng, Phys. Rev. D 38, 2869 (1988); H-Y. Cheng, Phys. Lett. B 219, 347 (1989).

[15] J. D. Vergados, On The Direct Detection of Dark Matter- Exploring all the signatures of the neutralinonucleus interaction, hep-ph/0601064

[16] J. Ellis , M. Karliner, Spin Structure Functions, CERH-TH 7072/93.

[17] M. T. Ressell et al., Phys. Rev. D 48, 5519 (1993); M.T. Ressell and D. J. Dean, Phys. Rev. C 56, 535 (1997).

[18] P. C. Divari, T. S. Kosmas, J. D. Vergados, and L. D. Skouras, Phys. Rev. C 61, 054612 (2000).

[19] A. K. Drukier, K. Freeze and D. N. Spergel, Phys. Rev. D, 33, 3495 (1986); J.I. Collar et al., Phys. Lett B 275, 181 (1992).

[20] J. D. Vergados, Phys. Rev. D 62, 023519 (2000).

[21] P. Sikivie, Phys. Rev. D 60, 063501 (1999).

[22] P. Sikivie, Phys. Lett. B 432, 139 (1998).

[23] J. D. Vergados, Phys. Rev. D 63, 06351 (2001).

[24] A. M. Green, Phys. Rev. D 63, 103003 (2001).

[25] G. Gelmini and P. Gondolo, Phys. Rev. D 64, 123504 (2001).

[26] C. Copi, J. Heo, and L. Krauss, Phys. Lett. B 461, 43 (1999).

[27] A. M. Green, Phys. Rev. D 66, 083003 (2002).

[28] A. S. Eddington, NRAS 76, 572 (1916).

[29] D. Merritt, A J 90, 1027 (1985).

[30] P. Ullio and M. Kamionkowski, JHEP 0103, 049 (2001). J.F. Navarro, C.S. Frenk and S.M. White, ApJ 462 (1996) 563.

[31] D. Owen and J. D. Vergados, Astrophys. J. 589, 17 (2003), astro-ph/0203923.

[32] J. Jeans, MNRAS 76, 70 (1915).

[33] S. H. Hansen, B. Moore, M. Zemp, and J. Stadel, JCAP 0601, 014 (2006), astro-ph/0505420.

[34] S. H. Hansen and B. Moore, New Astronomy 11, 333 (2006), ;astro-ph/041473.

[35] J. D. Vergados, Phys. Rev. D 58, 103001 (1998).

[36] J. D. Vergados, Phys. Rev. Lett. 83, 3597 (1999).

[37] H. C. Plummer, MNRAS 71, 460 (1911).

[38] M. H. von Ziepel, Ann. Obs. Paris 25, F1 (1908).

[39] D.N.C. Lin and J.E. Pringle, ApJ. 320, L87 (1987)

[40] J.D. Vergados, Direct SUSY Dark Matter Detection- Constraints on the Spin Cross Section, hep-ph/0512305

[41] N. Brouzakis and N. Tetradis, JCAP 0601, 004 (2006).

[42] The NAIAD experiment B. Ahmed et al, Astropart. Phys. 19 (2003) 691; hep-ex/0301039

B. Morgan, A. M. Green and N. J. C. Spooner, Phys. Rev. D 71 (2005) 103507; astro-ph/0408047 